\title{
LA ESTRUCTURA URBANA \\ COMO SOPORTE DE LA \\ CONTINUIDAD TIPOLÓGICA \\ TRAS UNA CATÁSTROFE:
}

\section{El Caso de San José de \\ Chamanga, Esmeraldas}

'Franco Tagliabuè Volonte, ${ }^{2}$ Renato D'Alençon Castrillón

\author{
'Dipartimento di Architettura e Studi Urbani, \\ Politecnico di Milano, Milano, ifdesign@tiscali.it \\ ${ }^{2}$ Centro de Desarrollo Urbano Sustentable, \\ CEDEUS y U. Católica, Santiago, Chile dalencon@uc.cl
}

Recepción / Received: 02, 11, 2016

Aceptación / Accepted: 28, 11, 2016

Publicado/Published: 08, 12, 2016

Resumen: San José de Chamanga, afectado por el devastador terremoto de magnitud $7.8^{\circ}$ en abril de 2016, como muchos otros poblados de la costa de Esmeraldas, sufrió la destrucción generalizada de su tejido edificado, hasta el punto de hacerse difícil localizar un punto de partida desde el cual iniciar la reconstrucción. En este artículo intentamos, a partir del trabajo en terreno, reconocer una estructura urbana que puede ser descrita e interpretada después del terremoto a pesar de la destrucción generalizada, para identificar en ella una cierta continuidad de la tipología urbano-arquitectónica del pueblo. El poblado está estructurado en torno a 5 áreas o bandas longitudinales: 1) la costa; 2) la calle principal; 3) la zona residencial; 4) el área comprendida entre las dos calles; y 5) la zona de la confluencia de todos los caminos. El reconocimiento de estas áreas permite elaborar nuevos mapas, reconocer el soporte urbano que antes recogió y puede volver a recoger lo que queda de la comunidad, la cultura y la economía locales.

Palabras claves: Chamanga, terremoto, tipología urbano-arquitectónica, reconstrucción 


\begin{abstract}
San José de Chamanga, affected by the devastating earthquake of magnitude $7.8^{\circ}$ in April 2016, like many other towns on the coast of Esmeraldas, suffered the widespread destruction of its built fabric, to the point of making it difficult to locate a starting point from which to initiate the reconstruction. In this article, we try to recognize an urban structure that can be described and interpreted after the earthquake in spite of widespread destruction, in order to identify a certain continuity of the urban-architectural typology of the town. The town is structured around 5 areas or longitudinal bands: 1) the coast; 2) the main street; 3) the residential area; 4) the area between the two streets; and 5) the area of the confluence of all roads. The recognition of these allows for the development of new maps, recognizing the urban support that was previously sustained and can again support what remains of the local community, culture and economy.
\end{abstract}

Keywords: Chamanga, earthquake, urban-architectural typologies, reconstruction.

\section{INTRODUCCIÓN}

San José de Chamanga es un pequeño pueblo de unos 5.000 habitantes situado en la costa Ecuatoriana del Pacífico, en la provincia de Esmeraldas, que se vio afectada por el devastador terremoto de magnitud 7.8 en abril de 2016. En Chamanga la comunidad, la cultura y la economía parecen no encontrar respuestas adecuadas para el mantenimiento de las actividades productivas que siempre han distinguido a toda la zona costera.

Mientras algunos de los mayores centros parecen haber encontrado una salida en el turismo de playa que trae nuevos flujos de dinero, al mismo tiempo desnaturaliza las características de estos territorios, pagando un alto precio a la colonización hotelera y de segundas residencias. En Chamanga, en tanto, muchas de las actividades han sido derivadas a un asentamiento de emergencia en las afueras (fig. 1).

La costa de Esmeraldas, tras la catástrofe, ha puesto al descubier- to una fuerte situación de contrastes que desde hace años está caracterizando la dinámica del desarrollo económico o la disminución de la población (INEC 2010).

El terremoto, como evento traumático, gatilla procesos introspectivos en sus componentes sociales, económicos, urbanos; inusuales y difíciles en condiciones normales (Brinckerhoff, 2005). Sin embargo, es necesa-

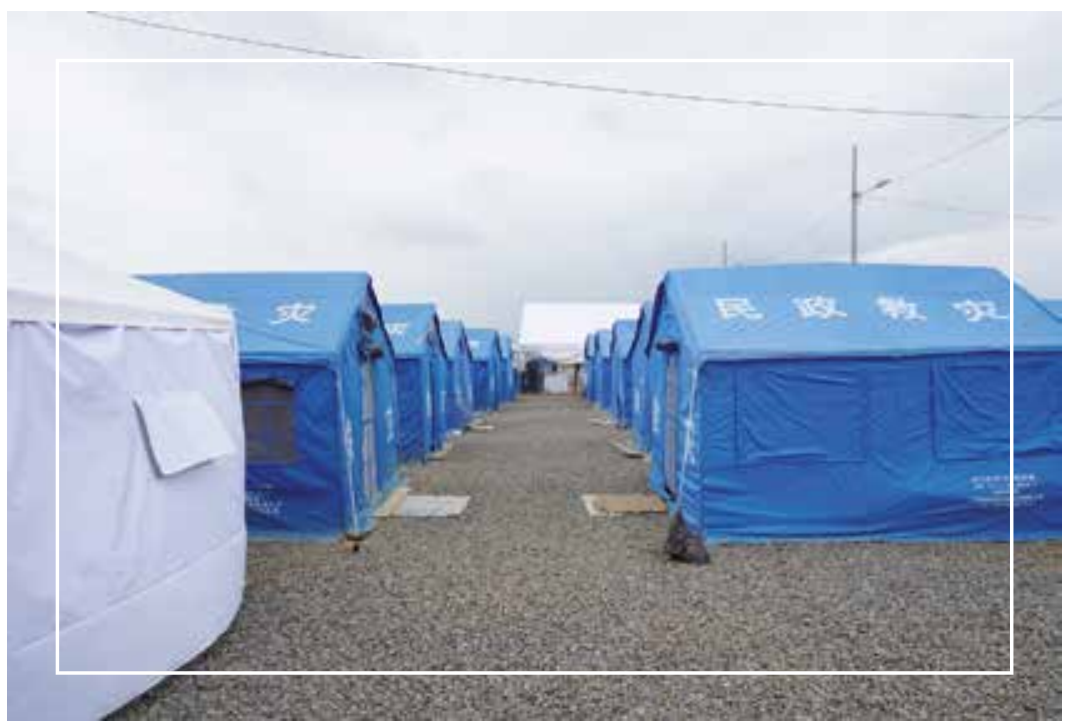

Figura 1. Campamento de Emergencia en las afueras de San José de Chamanga. Foto: R Moris 
rio un soporte físico que acoja estos procesos, en este caso casi totalmente destruido por el mismo sismo. En este artículo intentamos reconocer una estructura urbana que puede ser descrita e interpretada después del terremoto a pesar de la destrucción generalizada, para identificar en ella una cierta continuidad de la tipología urbano-arquitectónica del pueblo y el potencial que ella encierra para dar soporte a la reconstrucción.

\section{TERREMOTO Y OPORTUNIDAD}

Además de la trágica devastación, un terremoto representa un período crucial para el destino de las generaciones que habitan los lugares afectados durante las décadas y quizás siglos venideros. Casos notables, como los grandes incendios de Londres, de Chicago o de Hamburgo han dado paso a renovaciones urbanas de gran importancia en la historia de la ciudad (Benevolo, 1980). Al mismo tiempo, las nuevas oportunidades que se generan en los espacios dejados por el colapso y las nuevas expectativas van de la mano de las necesidades más apremiantes de la población, como viviendas, instalaciones de infraestructura y servicios, nuevas escuelas y tiendas que sean capaces de proveer condiciones para la vida en un grado suficiente de aceptabilidad o lo más cerca posible a la situación anterior al terremoto.

Pero lo que es quizás la oportunidad más grande es la reflexión sobre los errores y los valores del pasado que solo un evento catastrófico puede generar. Chamanga deberá ser capaz de reconocer la naturaleza y la calidad de la ciudad devastada por el terremoto. Tendrá que hacer un recuento acucioso de una realidad hecha de los daños que se sobreponen a las trazas de la compleja historia de los edificios y las personas que las habitaban y de todos los componentes que contribuyen, sobreponiéndose para formar la idea de la ciudad del pasado, del presente y del futuro (Fernández-Galiano, 2000). La documentación de las heridas del terremoto, mapeando una región, su cultu- ra, su economía y su forma, lo interpreta vislumbrando la posibilidad de su recuperación.

\section{RECONOCIMIENTO DEÁREAS}

El sistema urbano es relativamente simple y se caracteriza principalmente por cinco zonas longitudinales y paralelas entre sí que se acoplan perpendicularmente sobre el camino que viene desde el interior. Al igual que muchos pequeños poblados alrededor del mundo en los últimos años, resiste difícilmente el proceso de modernización y migración de la población hacia pueblos y ciudades más grandes.

El poblado está estructurado en torno a 5 áreas o bandas longitudinales: 1) la costa, donde se localizan las cabañas-palafito de los pescadores son del tipo tradicional; 2) la calle principal, donde hay edificios con tipos de constructivo predominante en hormigón armado; 3) la zona residencial situada en torno a un camino que remonta un desnivel de 12 metros, donde los edificios frente a la laguna se apoyan en un terreno inestable; 4) la parte del área comprendida entre las dos calles, donde una pendiente empinada está ocupada por un asentamiento informal; y 5) la zona de la confluencia de todos los caminos, en los que la catástrofe ha destruido el muelle de puerto y expandido el área terrestre.

La zona costera, con sus viviendas-palafito para los pescadores es, de hecho, la verdadera tipología tradicional de este poblado (fig 3). La banda que la constituye se forma a partir de una calle comercial que la limita. Las casas de pescadores son el sistema tipológico más reconocible en Chamanga, enraizadas en la tradición y la cultura de este pueblo. Al mismo tiempo es el enlace con el paisaje laguna y el tipo de habitación tradicional.

Las casas se agrupan en una estructura de clúster, directamente o con pequeñas desviaciones. Cada 


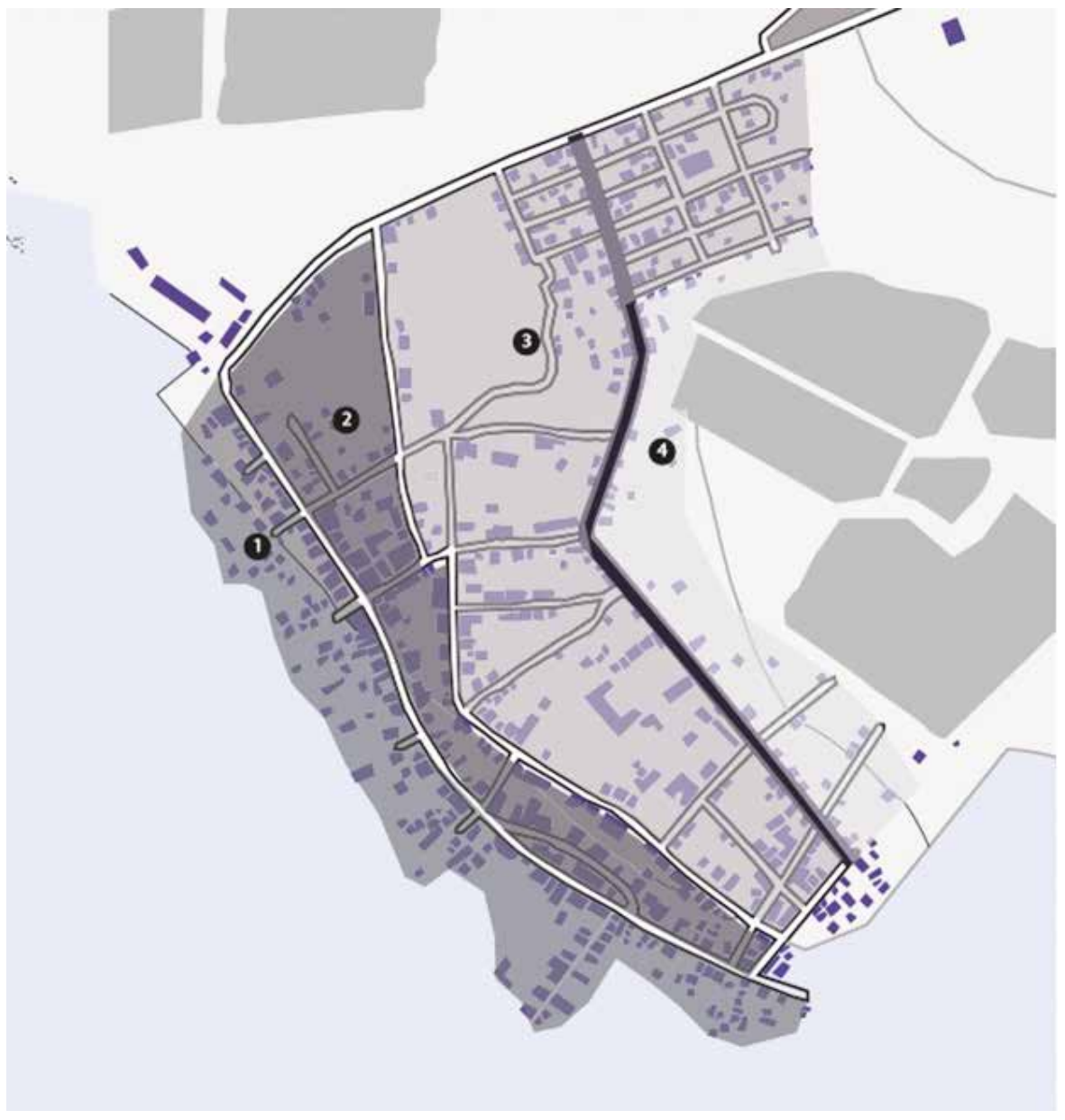

Figura 2. San José de Chamanga. Las zonas diferenciadas.

Dibujo: Equipo Universidad Tecnológica Equinoccial. Workshop WINAREQ 2016

La cubierta es generalmente a dos aguas de chapa metálica, con pequeñas proyecciones 0 sencillamente sin ellas.

Las paredes perimetrales son, básicamente, listones de madera de alrededor de 20 centímetros dispuestas de forma horizontal, aunque en muchos casos se pueden encontrar otros materiales, incluso láminas de metal corrugado o enyesado.

Tales diferencias parecen ser de carácter totalmente espontáneo, presumiblemente derivadas de la disponibilidad y el coste del material. Pero es posible que haya una necesidad de una representación y la diferenciación entre las propiedades de los habitantes. Las puertas y ventanas están

muelle agrupa de 5 a 15 viviendas cuyo acceso común se conecta directamente a la línea de costa. Las plataformas que se podría llamar propias de las casas individuales están equipadas con escaleras que permiten llegar directamente a las pequeñas embarcaciones amarradas que flotan abajo en el agua. En muchos casos se encuentra un pequeño espacio abierto $y$ cubierto destinado al descanso, a menudo con una hamaca, una característica esta última que se repite en la mayoría de los hogares de Chamanga. La diferencia es que en las casas de los pescadores todo parece más pequeño. hechas principalmente de madera, aunque a menudo las aberturas están totalmente desprovistas de marcos vidriados y en general las ventanas son de tamaño pequeño.

La calle principal, que es el principal eje comercial, para los habitantes del pueblo antes del terremoto. Está separada del borde costero principal al estar flanqueda por edificios con tipo predominante de construcción en hormigón armado, que, mal construido, es el que ha sufrido los más grandes daños (fig. 4), 
revelando al mismo tiempo la oportunidad de recuperar el borde.

Aunque en los últimos años las casas de hormigón armado de la franja interior se consideraron un objetivo para las personas que aspiraban a un estatus social más alto, son estas las que han mostrado una debilidad, constructiva y de sistema, que se ha resuelto con su sustitución implacable: cemento mal construido, tierra mezclada con áridos inadecuados y, a menudo, con agua de mar, con refuerzos de acero oxidados por las fracturas generadas por el terremoto que muestran los cálculos aproximados y en ocasiones utilizados al azar, que no fueron capaces de ofrecer ese grado de modernidad al que sus constructores aspiraban, como si la mano del destino hubiera elegido por sí sola, inexorablemente, los edificios que levantados con demasiada rapidez- no pertenecen al verdadero cuerpo de la ciudad, destruyéndolos.

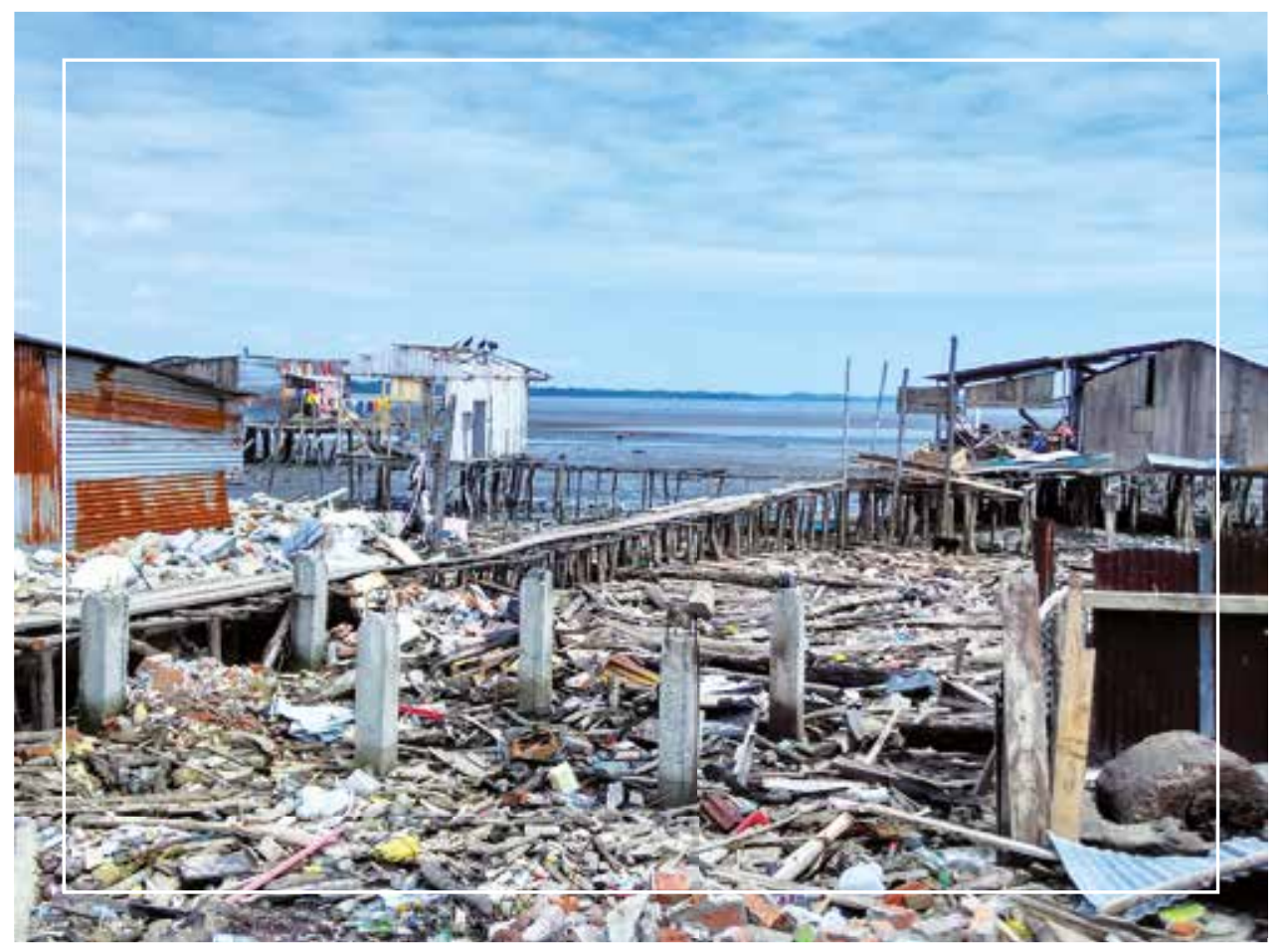

Figura 3. La zona costera después de la catástrofe. Foto: R D'Alencon
El área residencial está estructurada por un camino que se eleva a una altura máxima de unos 12 metros: aquí los edificios situados en el borde (fig. 5) se apoyan sobre un terreno muy frágil para sostenerlos, pero disfrutan en muchos casos de una agradable vista desde la altura, hacia la laguna y hacia las suaves pendientes del interior. Si se considera asegurar la parte alta de la trama urbana, será necesario intervenir con obras de ingeniería para la consolidación de la tierra y esto podría representar una buena oportunidad para una lectura de estas intervenciones como un nuevo espacio público recorrible longitudinalmente de carácter peatonal: el verdadero espacio alternativo (aunque en el mismo sentido) al sistema de calles vehiculares existente.

En la actualidad, la mayoría de los edificios no presentan características dimensionales únicas aunque a menudo consisten en un solo ambiente (aproximadamente 4 a $5 \mathrm{~m}$ de lado), en la mayoría de los casos totalmente libres de tabiques interiores. Se pueden encontrar también módulos con dimensiones más grandes, rara vez con una medida mayor que el doble de la anterior. La planta es rectangular con raras excepciones que presentan adiciones y acumulaciones. El tipo es sobre pilotes con estructura de madera, con aproximadamente un metro ochenta sobre la superficie de la laguna y están 


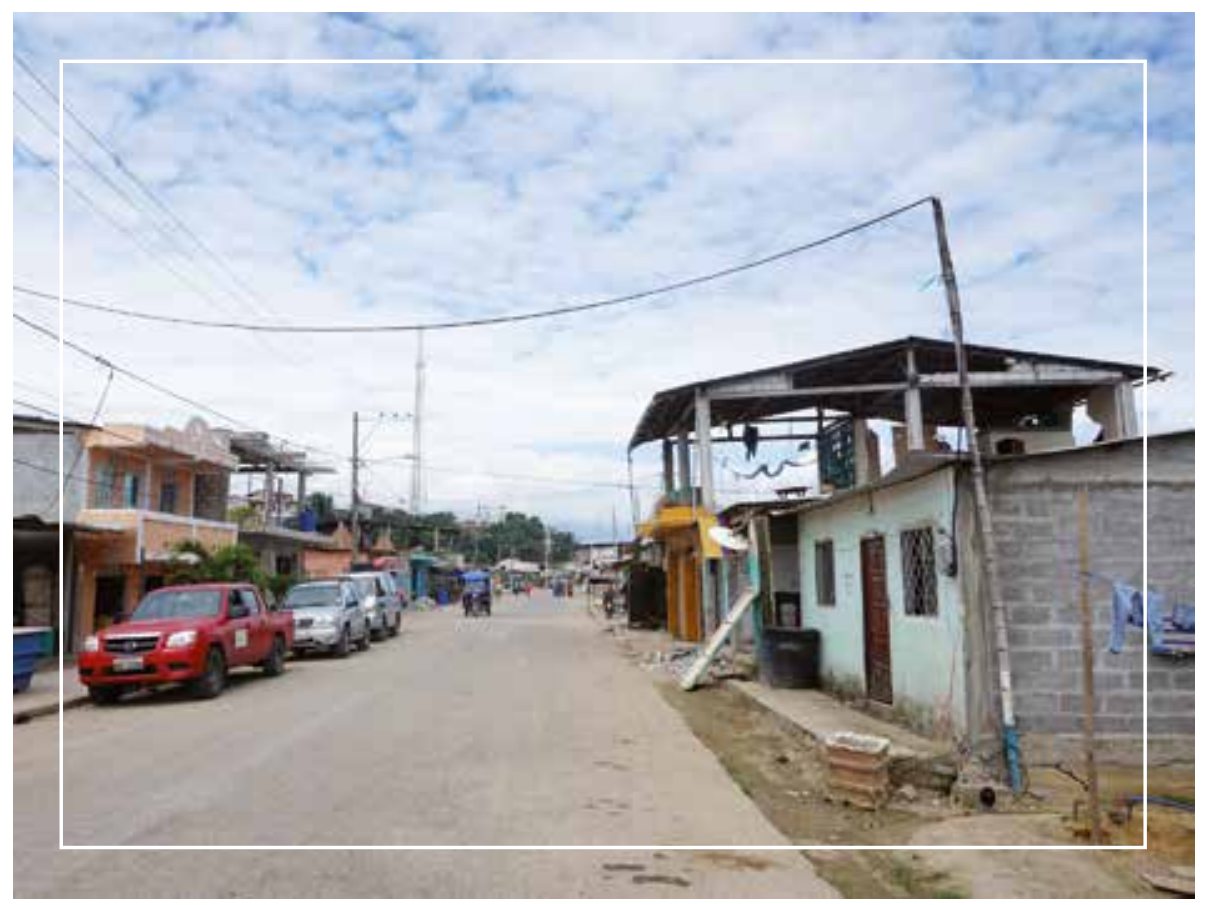

Figura 4. La calle principal, separada del borde por la densificación de las fachadas. Foto: $R D^{\prime}$ Alencon

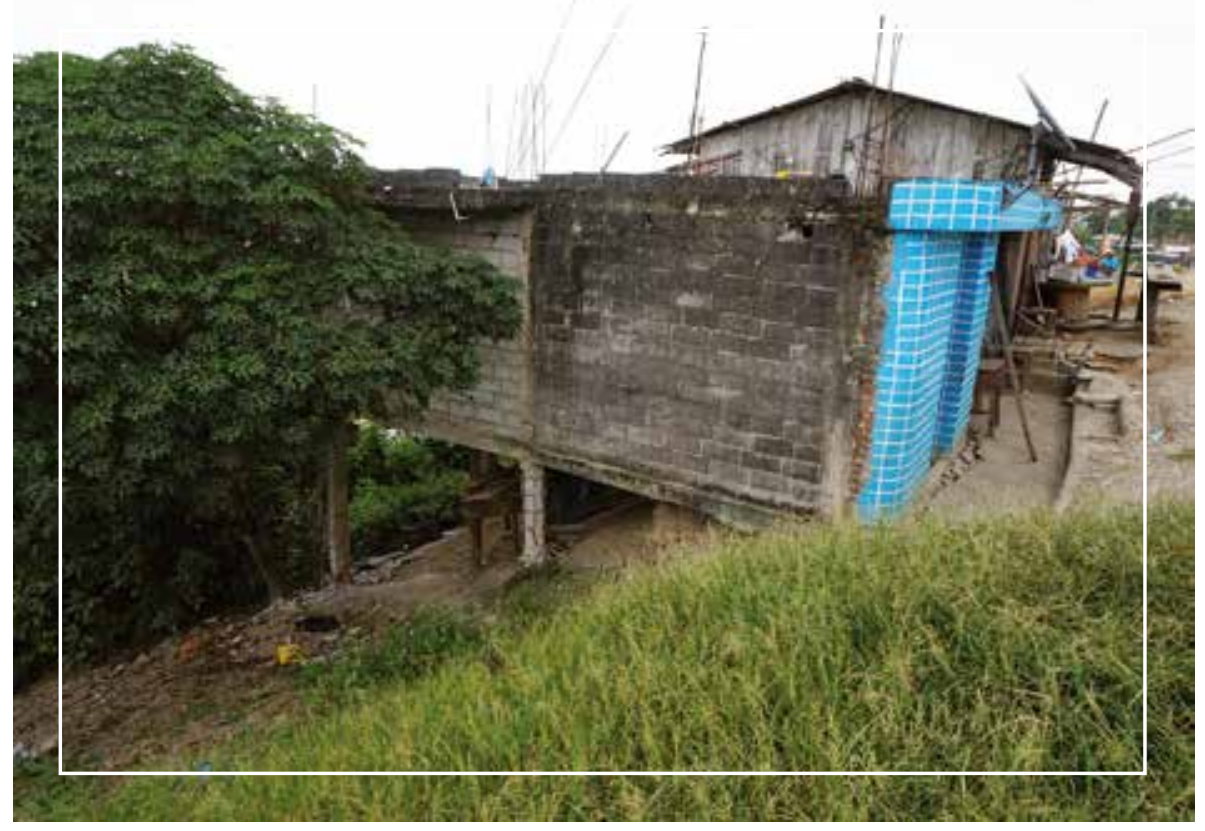

Figura 5. Las casas en la parte alta de la ciudad, colocadas sobre la pendiente. Foto: R Moris conectadas al continente por un complejo sistema de muelles y pasarelas perpendiculares a la línea de tierra firme. Se trata de estructuras autoconstruidas de carácter informal o espontáneo.

\section{La banda entre los dos} caminos es una pendiente empinada, ocupada de manera informal por edificios, mientras que las calles de Chamanga desembocan en una amplia explanada de tierra ampliada (fig. 6), por la catástrofe que destruyó el muelle del puerto.

La banda que corre paralela entre las dos calles, alta y baja, está ocupada de manera informal por extensiones de las casas de abajo, o deja el espacio vacío y descuidado ningún papel evidente en la ciudad. Sin embargo, la mayoría de los espacios públicos más connotados, pequeñas escaleras que terminan en lugares igualmente mínimos de observación en el alto, cruzan la transversal $y$ unen las dos partes del pueblo. 


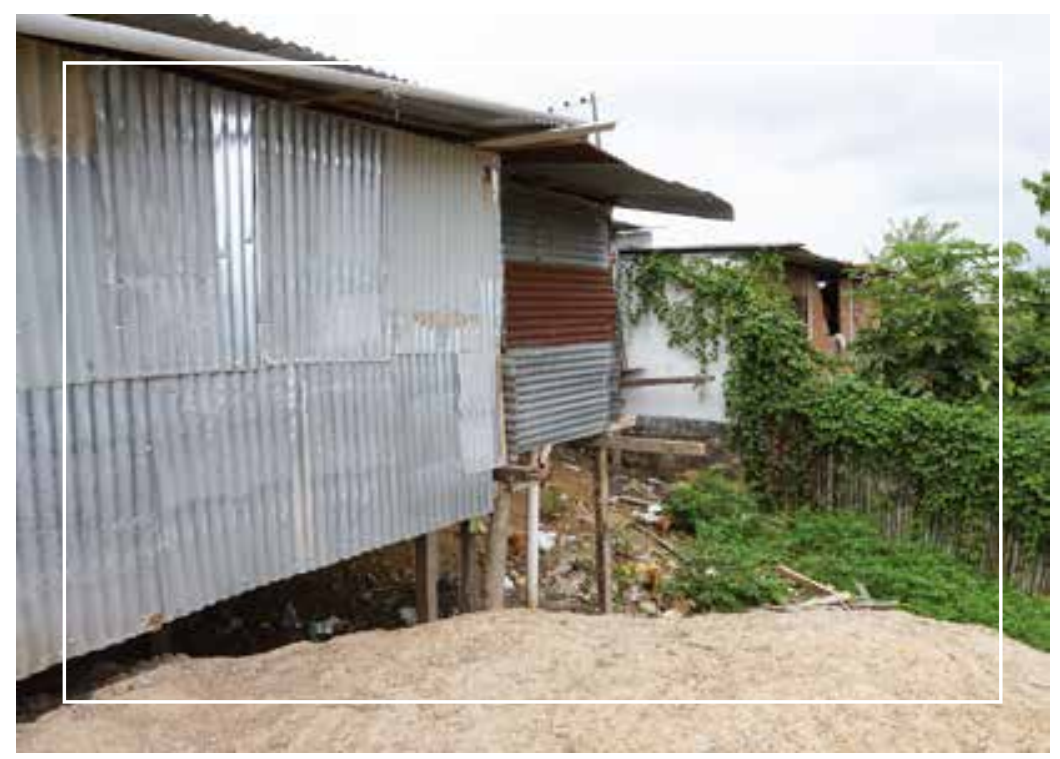

Figura 6. Las casas de la franja entre dos caminos, predominantemente informales. Foto: F. Tagliabue
Cada una de las cinco bandas presentan el estado actual de las cosas, diferentes y a la espera de sus escenarios específicos. Pero si pueden articular unos con otros, se convierten en complementarios, y la nueva ciudad será más fuerte que la existente, los problemas se transforman en oportunidades y encontrar una salida difícil de imaginar sin la ocurrencia del sismo.

Este tipo de vivienda ha sufrido el menor número de daños del terremoto debido a las propiedades de la estructura de madera con buena elasticidad $y$, por tanto, una mayor capacidad de resistir a la acción sísmica. Es probable que los palafitos profundos hayan contribuido a la estabilidad de las viviendas.

\section{DISCUSIÓN:}

\section{DAÑOS Y POTENCIALES}

Si muchos de los edificios que componen el centro del poblado ciudad no parecen merecer recuperación y parece casi seguro su reubicación en zonas más seguras y en formas más apropiadas (tal vez un nuevo asentamiento que ya está naciendo en el campo de emergencia), la estructura urbana que se ha descrito anteriormente constituye a su vez, de hecho, la "naturaleza de la ciudad", que se deriva de su historia (Ortega y Gasset, 1970). Llevar adelante una idea que no pasa por la confirmación o la reinterpretación de esta implantación urbana, modificado consistentemente en razón de la lógica de la seguridad y la prevención, será como borrar el alma de una ciudad y privarla de su futuro.
Por lo tanto, la situación no es particularmente crítica, aunque la condición de los edificios no parece particularmente buena debido a las técnicas de construcción, la mala calidad de los materiales utilizados, y especialmente un mantenimiento muy precario.

Las excepciones son los edificios situados en el sureste, cerca del muelle, donde se produce el mayor daño a viviendas de esta tipología. Aquí el fenómeno de la licuefacción del suelo ha determinado el colapso de la mayoría de las casas. La zona parece haber sido intervenida por unos con un relleno de tierra en el área entre las casas y el muelle.

Mientras que para todas las casas de Chamanga no se puede reconocer un tipo real, si no en el mejor de los casos algunas características tipológicas recurrentes, las viviendas de los pescadores caracterizan el paisaje 
habitado del pueblo de forma inequívoca. Ocupan una banda de territorio urbano particularmente característico, sujetos a la retirada de las mareas, de manera que durante algunos intervalos de tiempo bajo las viviendas se detecta la presencia de agua, mientras que a veces la parte inferior se mantiene en condiciones secas con las barcas descansando directamente sobre la superficie de la laguna.

No solo el paisaje está caracterizado por la presencia de la laguna, hecho de suyo sustancial desde el punto de vista arquitectónico, sino que a su vez esta condición contribuye a la singularidad del conjunto poblado.

Sería, por tanto, propicio un tipo de regulación arquitectónica de estos edificios, destinada a preservarlos y consolidarlos tipológicamente. No se trata solo de la preservación de edificios tradicionales, sino más bien entender y reforzar el alto potencial para un proyecto de desarrollo de la economía local. Parece obvio que una de las metas más razonables para fines turísticos es transmitir la imagen de Chamanga que más la distingue, capaz de generar sugerencias importantes de un determinado tipo de turismo, especialmente practicado en los últimos años, que se podría llamar "turismo ecológico".

El interés del usuario en este caso es principalmente cultural, el objetivo es conocer en profundidad las tradiciones y costumbres de las personas que habitan en un territorio; entrar en contacto con una realidad, una forma de vida que solo se manifiesta aquí con una perfecta integración con el paisaje.

Se considera que es importante mantener la función actual, permitiendo y favoreciendo la elevación del estándar de calidad de la vivienda.

\section{CONCLUSIÓN}

Aunque el estado de conservación es precaria, esta condición no debe malinterpretarse con un valor negativo absoluto. Por el contrario su plena recuperación, combinado con un cuidadoso trabajo de actualización, realmente puede ser el motor del desarrollo de nuevas economías en condiciones de otorgar un futuro en este pequeño pueblo costero.

Esta intervención representa una buena oportunidad de construir una conexión transversal entre los muelles con el fin de mejorar la eficiencia de la red. La pasarela resultante sería de hecho un margen para la construcción de la calle y una ocupación compatible y necesaria para poner orden en esa franja de tierra no bien definida entre la laguna y los edificios cercanos a la calle principal, que incluso antes del terremoto resultó saturada de escombros y residuos.

Una intervención así estructurada no solo irá en beneficio de las condiciones de habitabilidad de los pescadores, sino que debe respetar el sistema de lagunas ecológicas y las condiciones generales de salud de la población.

Algunos de los edificios, u otros nuevos diseñados con la misma matriz tipológica, además de ofrecer información y servicios turísticos, podrían ser dedicados a la infraestructura de las actividades pesqueras, como almacenes de herramientas, y de refrigeración común, así como pequeñas terminales de venta. Solo por formas asociadas de uso de estas áreas, como se ha demostrado por algunos ejemplos de regeneración en otras partes del continente, se puede pensar en el futuro de estas pequeñas economías, pero representan la gran historia de toda una zona. 


\section{REFERENCIAS}

- Benevolo, L. (1980). The History of the City. Boston: MIT Press.

- Brinckerhoff J. (2005). De la nécessité des ruines et autres sujets. Editions du Linteau.

- GAD de Chamanga. Parroquia de San José. En línea (acceso octubre 2016): http://www.chamanga.gob.ec/

- Instituto Nacional de Estadística y Censos INEC. (2010). Censo de Población y Vivienda, Quito, Ecuador.

- Ortega y Gasset, J. (1970). Historia como Sistema. Madrid: Ediciones de la Revista de Occidente.

- Secretaría de Planificación y Desarrollo SENPLADES. (2015). Consultora Coral. Plan de Desarrollo Territorial de San José de Chamanga. Quito.

- Tagliabue Volontè, F., y N. Bassoli. (2016). Maggioli, Sant'Arcangelo di Romagna - StemProcedure Post-Earthquake Regeneration Strategies.

- Ligi, G. (2009). Antropologia dei disastri. Roma: Laterza.

- Fernández-Galiano, L. (2000). Terremoto e Terapia. Lotus 104, pag. 44-47, Milano, 2000. 